\title{
Propiedades psicométricas de la Escala Breve Multidimensional de Satisfacción con la Vida para Estudiantes (BMSLSS) en población infantil chilena (10-12 años)*
}

Psychometric Properties of the Brief Multidimensional Students' Life Satisfaction Scale (BMSLSS) in Chilean Students (10 - 12 years old)

Recibido: 20 de noviembre de 2013 | Revisado: 21 de junio de 2014 | Aceptado: 21 de junio de 2014

\author{
JAIme Alfaro I. ** \\ JAVIER GUZMÁN P. \\ Catalina García G. \\ DAVID SIRLOPÚ D. \\ LORRAINE GAUDLITZ R. \\ JUAN OYANEDEL S. \\ Universidad del Desarrollo, Santiago, Chile \\ Juan Carlos Oyanedel Sepúlveda*** \\ Universidad Santiago de Chile, Santiago, Chile
}

doi: 10.11144/Javeriana.upsy14-1.ppeb

Para citar este artículo: Alfaro, J., Guzmán, J., García, C., Sirpolú, D., Gaudlitz, L., \& Oyanedel, J.C. (2015). Propiedades psicométricas de la Escala Breve Multidimensional de Satisfacción con la Vida para Estudiantes (BMSLSS) en población infantil chilena (10-12 años). Universitas Psychologica, 14(1), 29-42. http://dx.doi.org/10.11144/Javeriana. upsy14-1.ppeb

* Artículo original resultado de investigación

*** Facultad de Psicología. Ave. La Plaza No680, Las Condes. Santiago, Chile. Correos electrónicos: jalfaro@udd.cl, javierguzman@udd.cl, catalinagarcia@udd.c, dsirlopu@udd.cl, lgaudlitzr@udd.cl

**** Facultad de Administración y Economía. Ave. Libertador Bernardo O'Higgins 3363, Estación Central. Santiago, Chile. Correo electrónico: juan. oyanedel@usach.cl

\section{RESUMEN}

Este trabajo analiza las propiedades psicométricas de la escala BMSLSS de Seligson, Huebner y Valois (2003) en niños y niñas chilenos. Se aplicó a 1096 estudiantes de 10, 11 y 12 años. Se realizaron análisis de confiabilidad, análisis factorial exploratorio (EFA) y confirmatorio (CFA) y correlación ítem con puntaje total. Los resultados exhiben una aceptable confiabilidad interna de la escala $(\alpha=0.7)$, un solo factor en la estructura factorial de la escala y adecuada correlación ítem con puntaje total (entre 0.55 y 0.73). El estudio fundamenta el uso de BMSLSS en Chile en población infantil y aporta herramientas de medición de indicadores sociales para la infancia en países en vías de desarrollo de habla hispana.

Palabras clave

bienestar subjetivo en la infancia; satisfacción con la vida; BMSLSS; infancia; propiedades psicométricas

\section{A B S T R A C T}

This paper analyzes the psychometric properties of the Brief Multidimensional Students' Life Satisfaction Scale -BMSLSS (Seligson, Huebner and Valois, 2003) in Chilean children. 1096 Chilean students of 10, 11 and 12 years-old participated in the study. Reliability analysis, exploratory factor analysis (EFA), confirmatory factor analysis (CFA) and item correlation with total score were performed. Results show acceptable internal reliability of the scale $(\alpha=0.7)$, a single factor structure of the scale and appropriate item correlation with total score (between 0.55 and 0.73 ) This study supports the use of the BMSLSS in children in Chile in the school context, and also provides measurement tools for social indicators for children in Spanishspeaking developing countries.

Keywords

children's subjective well-being; life satisfaction; BMSLSS; children; psychometric properties 


\section{Bienestar subjetivo y satisfacción con la vida}

La investigación en calidad de vida ha sido conceptualizada desde dos perspectivas: objetiva y subjetiva. El punto de vista objetivo se centra en las condiciones externas que contribuyen a la calidad de vida, tales como los niveles de ingresos, calidad de la vivienda, redes de amistad y el acceso a los servicios de salud. En cambio, la perspectiva subjetiva refiere a los juicios internos de los individuos de su calidad de vida. Estos juicios o sentencias se refieren a satisfacción con la vida, en general y/o ámbitos específicos de la vida (por ejemplo, la satisfacción con amigos, experiencias escolares, familia). De tal manera que, si bien las circunstancias objetivas de vida son importantes en la determinación de la calidad de vida de los individuos, las interpretaciones y evaluaciones subjetivas de sus experiencias son más cruciales (Valois, Zullig, Huebner, \& Drane, 2004a).

La noción de bienestar subjetivo está estrechamente ligada a la satisfacción con diferentes aspectos de la vida y con una evaluación más integral, denominada satisfacción global con la vida (Figuer et al., 2004). Para Campbell, Converse y Rodgers (1976) estos procesos se pueden medir a través de preguntas de satisfacción, de forma tal que, según Cummins, Eckersley, Van Pallant, Vugt y Misajon (2003), la esencia del bienestar subjetivo está referida mayormente a las evaluaciones que hace una persona sobre la satisfacción personal con su vida.

Diener (2006) considera que el bienestar subjetivo refiere a varios tipos de opiniones, buenas o malas, que la gente tiene sobre su vida, incluyendo evaluaciones cognitivas, como la satisfacción en el trabajo y la vida, el interés y el compromiso y las reacciones afectivas frente a acontecimientos de la vida como alegrías y tristezas. Petito y Cummins (2000) entienden el bienestar en términos de la evaluación individual que una persona hace de su calidad de vida, el cual está formado por dos componentes: el primero es la satisfacción de vida, lo que implica una evaluación cognitiva, y el segundo es emocional, que implica sentimientos y estado de ánimo.

De acuerdo con la opinión de Diener (1984), hay un fuerte consenso sobre las tres característi- cas básicas del bienestar subjetivo: (1) se construye a partir de las experiencias de cada persona y de la percepción y evaluación de tales experiencias, (2) incluye dimensiones positivas y no solamente la ausencia de negativas y (3) incluye algunas evaluaciones globales de la vida, que por lo general se conoce como "satisfacción con la vida".

Delimitando aún más esta noción, se entiende que el bienestar subjetivo está constituido por tres dimensiones interrelacionadas: los afectos positivos, los afectos negativos y la satisfacción con la vida (Diener \& Suh, 1997; Pavot \& Diener, 1993). Otros autores consideran también el componente de felicidad (Casas, 2010a).

En este marco, específicamente la satisfacción con la vida es entendida como una evaluación global de la persona sobre la calidad de su vida ante sus circunstancias (Seligson, Huebner, \& Valois, 2003), que da cuenta del grado en que evalúa positivamente su vida como globalidad (Veenhoven, 1994) o con referencia a dominios específicos como la familia, amigos o la escuela (Huebner, 2004; Seligson, Huebner, \& Valois, 2005), relativa a un estado presente (Veenhoven, 1994).

La relevancia de esta noción tiene relación con que los datos de satisfacción con la vida que permiten calcular la calidad de vida "realizada" en un país o categoría social, son útiles en la validación empírica de las condiciones necesarias para el vivir bien, y con ello informan acerca de qué es una buena sociedad (Veenhoven, 1994). Además, permite evaluar los efectos en la calidad de vida de las políticas aplicadas, siendo de esta manera de gran importancia para trazar estrategias de desarrollo social (Veenhoven, 2002). De este modo, el estudio y desarrollo de medidas de satisfacción con la vida proporciona una importante información para la prevención, la detección precoz y la intervención en diversas poblaciones en condiciones de riesgo (Seligson et al., 2005).

\section{Bienestar subjetivo en la infancia}

La disponibilidad de estudios sobre el bienestar de niños, niñas y adolescentes dista mucho de alcanzar la cantidad de resultados que ofrece la 
investigación con adultos (Casas, 2010a; Casas et al., 2012; Gademann, Schonert-Reichl, \& Zumbo, 2010; Huebner, 2004; Huebner \& Gilman, 2002; Seligson et al., 2003; Tomyn \& Cummins, 2011) tanto cuantitativa como cualitativamente (Casas, 2010a). A la vez, los pocos datos disponibles sugieren que durante las etapas más jóvenes de la vida el funcionamiento del bienestar tiene su propia idiosincrasia evolutiva a lo largo de los años, diferenciándose por tanto según edad (Casas, 2010a).

El estudio del bienestar subjetivo en la infancia $\mathrm{y}$ adolescencia es un campo que requiere mayor desarrollo y avance investigativo (Casas, 2010b). Según señalan Pollard y Lee (2003), gran parte de los trabajos producidos referirían de manera principal a población en condiciones médicas crónicas, concentrándose en el campo de la pediatría, principalmente la investigación de la calidad de vida en niños con enfermedades crónicas.

También se requiere avance en el desarrollo de instrumental para la medición del bienestar subjetivo en la infancia y adolescencia, tanto para aumentar la comprensión de sus correlatos como medir su estado, y de igual forma guiar acciones tendientes a su elevación y mejora, complementariamente a una aproximación centrada en la evaluación de las conductas problemáticas o dimensiones patológicas de estos (Gademann et al., 2010). Además, permitiría conocer los factores relacionados con las diferencias individuales en la percepción del bienestar y sus secuelas (Huebner \& Gilman, 2002; Seligson et al., 2003, 2005), así como la identificación de subgrupos de riesgo para garantizar la adecuada prestación de apoyo y recursos a los que lo necesitan (Tomyn \& Cummins, 2011).

En particular, se requiere desarrollar medidas comparativas nacionales y transnacionales y de grupos o entornos culturales diversos (Casas, 2010a; Casas et al., 2012; Siyez \& Kaya, 2008), pues existen, hasta ahora, escasas publicaciones con prueba empírica de los resultados obtenidos con diferentes instrumentos aplicados a infancia y adolescencia en diferentes países o contextos culturales, siendo la mayoría realizados en el mundo anglosajón, con algunas excepciones (Casas et al., 2012).
Sumado a ello, el ámbito de investigación en bienestar subjetivo en población infantil y adolescente (y también adultos) no se ha producido de manera significativa en países en vías de desarrollo, tal cual reportan Lau, Cummins y McPherson (2005), Tiliouine, Cummins y Davern (2006), Siyez y Kaya (2008), Webb (2009) y Yiengprugsawan, Seubsman, Khamman y Lim (2010). Se conocen reportes con base en el Índice de Bienestar Personal (PWI) aplicados solo en países en vías de desarrollo, tales como Tailandia (Yiengprugsawan et al., 2010), Argelia (Tiliouine et al., 2006) o con la BMSLSS en Turquía (Siyez \& Kaya, 2008). Uno de los avances en América Latina fue realizado en 2010 mediante el estudio transcultural sobre bienestar subjetivo en Chile, Brasil, Argentina y en España con adolescentes de 12 a 16 años (Casas et al., 2011).

En Chile, además, se han validado escalas en adolescentes y estudiado aspectos que predominan en la visión que los adolescentes tienen sobre su satisfacción global con la vida (Alfaro, Valdenegro, \& Oyarzún, 2013), analizado psicométricamente instrumental como la escala Ryff (versión española) en población adolescente respecto de su bienestar psicológico (Gallardo Cuadra \& Moyano Díaz, 2012). En adultos mayores, también se cuenta con instrumental adaptado y validado preliminarmente como la escala de felicidad de MUNSH (Moyano Díaz, Flores Moraga, $\&$ Soromaa, 2011). Adicionalmente, se ha estudiado el bienestar subjetivo, utilizando la Escala de Satisfacción con la Vida de Diener, analizando sus propiedades psicométricas en chilenos entre 18 y 65 años (Vera-Villarroel, Urzúa, Pavez, CelisAtenas, \& Silva, 2012). Pese a estos aportes sustantivos en el tema, no se cuenta con estudios de bienestar en población infantil, así como tampoco con el desarrollo de instrumentos validados para este grupo de edad.

Pese a estos desarrollos, en América Latina sigue siendo necesario avanzar de manera sistemática en el conocimiento sobre calidad de vida de los niños y adolescentes, y en particular sobre su calidad de vida en dimensiones subjetivas, según sus diversos contextos y la diversidad de situaciones colectivas de la región (Aguado, 2009). 
Según investigadores internacionales del campo, tales como Ben-Arieh (2008), Bradshaw y Richarson (2009), Bradshaw y Lau (2010) y Casas (2010b), existe la necesidad de crear indicadores sobre infancia que, entre otras acciones, contemplen el uso de indicadores de resultado y mediciones directas del bienestar más que el uso de indicadores indirectos; tengan como unidad de análisis y de información al niño (en vez de los padres, la familia o el hogar); den prioridad a indicadores del bienestar actual de los niños, más que a indicadores de futuro bienestar o realización como adultos -diagnosticar qué ocurre con sus condiciones de vida en el presente- y usen indicadores sobre sus sentimientos y su vida.

\section{Satisfacción con la vida y problemas sociales}

El bienestar subjetivo en sus distintas acepciones, se ha relacionado con la integración social de las personas, la salud y la organización comunitaria. Por otra parte, las medidas de satisfacción global con la vida y sus diferentes componentes, al ser combinadas con escalas clínicas, han resultado ser buenos predictores de salud mental en población infantil y adolescente. (Huebner, Suldo, \& Valois, 2003).

Específicamente, el estudio de la satisfacción con la vida permite determinar riesgos en dimensiones tanto psicológicas como sociales, tiene una valiosa capacidad predictiva respecto de estados patológicos, así como en la capacidad de manejo de eventos vitales estresantes y sus efectos en problemas de comportamiento (Puente Díaz \& Cavazos Arroyo, 2013). Por último, contribuye con información clave para intervenciones sociosanitarias como la prevención y detección precoz, en diversas poblaciones en condiciones de riesgo.

Las medidas de satisfacción o insatisfacción vital son útiles también para propósitos clínicos. Así, algunas investigaciones en niños reportan que los puntajes bajos en satisfacción, ofrecen información relevante sobre el bienestar, especialmente en niños que no presentan psicopatologías de base. Mediciones tradicionales de evaluación psicológica parecen dar a entender que los niños que no presentan psicopatología tendrían necesariamente un adecuado nivel de bienestar subjetivo, lo que según la evidencia pareciera ser engañoso, en cuanto los niños que no presentan signos de patología clínicamente relevante (por ejemplo, síntomas depresivos) no necesariamente experimentan niveles elevados de bienestar subjetivo. Como resultado, algunos grupos de niños pueden pasar desapercibidos por los profesionales de salud mental, con consecuencias negativas para su bienestar y el desarrollo de medidas preventivas (Seligson et al., 2005).

La importancia de la investigación en satisfacción con la vida se ha demostrado en estudios que indican que los adolescentes que no están satisfechos con la vida son más propensos a abusar de las drogas, se involucran en conductas violentas y agresivas y con más frecuencia toman riesgos sexuales (por ejemplo, las relaciones sexuales sin protección) (Paxton, Valois, Huebner, $\&$ Drane, 2006).

Se cuenta con evidencia respecto de su utilidad predictiva, en relación con que la medición de la satisfacción con la vida (por medio específicamente de la BMSLSS) ha demostrado asociación con conductas de riesgo en los jóvenes. Específicamente, en adolescentes, la BMSLSS ha demostrado utilidad predictiva en relaciones simultáneas, negativas con el alcohol, el tabaco y el consumo de drogas ilegales, así como con sus comportamientos de riesgo violentos y agresivos, tales como la lucha física y con armas (Huebner et al., 2003).

La evaluación de la satisfacción vital puede ser útil también en cuanto los estudios con adolescentes muestran asociaciones significativas entre esta dimensión y comportamientos de riesgo importantes, tales como la asunción de riesgos sexuales, ideación y comportamiento suicida (Valois et al., 2004a), problemas adaptativos relacionados con la alimentación (Valois, Zullig, Huebner, \& Drane, 2003), y el ejercicio y la actividad física (Valois, Zullig, Huebner, \& Drane, 2004b). Otra investigación con adolescentes indica que la satisfacción con la vida en general desempeña un papel mediador en la relación entre los eventos estresantes de la vida y conductas internalizantes 
en adolescentes (McKnight, Huebner, \& Suldo, 2002), y un papel moderador de la relación entre los eventos estresantes de la vida y conductas externalizantes en adolescentes (Huebner, Suldo, Valois, \& Drane, 2006).

Visto lo anterior, en términos generales, la satisfacción con la vida está integralmente vinculada a una amplia gama de indicadores físicos, mentales, de rendimiento escolar, emocionales y sociales de funcionamiento humano, lo que sugiere que esta noción da cuenta de una fortaleza psicológica clave (Jiang, Huebner, \& Hills, 2013), por lo que las medidas de bienestar subjetivo y satisfacción con la vida son una herramienta relevante que permite extender el alcance de los indicadores de bienestar más allá de la primacía de los indicadores objetivos utilizados en la toma de decisiones gubernamentales (Valois et al., 2003).

\section{Escala Breve Multidimensional de}

Satisfacción con la Vida para estudiantes

La Escala Breve Multidimensional de Satisfacción con la Vida para Estudiantes (BMSLSS) de Seligson et al. (2003) fue desarrollada en respuesta a un creciente interés en la evaluación, supervisión y promoción de los aspectos positivos del bienestar de los individuos, orientada a la medición de la satisfacción con la vida (Huebner, Antaramian, Hills, Lewis, \& Saha, 2011). Está compuesta de seis ítems y considera la satisfacción en ámbitos de familia, amigos, escuela, sí mismo, barrio y con la vida en general. Por lo anterior, resulta una escala que facilita la implementación de estudios a gran escala, evaluaciones longitudinales y medidas de tamizaje breve (Seligson et al., 2003).

En distintos niveles educativos, se han obtenido propiedades psicométricas adecuadas. La escala reporta un alpha de Cronbach de la puntuación total de la BMSLSS (en su versión de cinco ítems) de 0.75 para estudiantes de middle school y de 0.81 para los estudiantes de high school (Seligson et al., 2003). En términos generales los coeficientes reportados van desde 0.8 hasta 0.85 y se observa un solo factor significativo que explica la mitad de la varianza (Huebner et al., 2003).
La validez de la escala BMSLSS con adolescentes ha sido examinada en diversos estudios mediante procedimientos de análisis factorial exploratorio (EFA) y confirmatorio (CFA). Los resultados revelaron una fiabilidad aceptable, consistencia interna, validez de criterio y validez de constructo de la puntuación total. La validez convergente se ha observado entre las puntuaciones del BMSLSS y medidas alternativas de satisfacción con la vida, como la Escala de Satisfacción de Vida Estudiantil (Huebner, 1991). Así, también se han encontrado correlaciones negativas entre la BMSLSS y algunos indicadores de salud mental (por ejemplo, la depresión y la ansiedad; Jiang et al., 2013).

Los resultados de la BMSLSS entre los adolescentes de varias razas, sexo y grupos de edad indican que su puntuación total es una medida del desarrollo positivo de la juventud que no está influenciada por las diferencias demográficas (Huebner et al., 2003). Sí se observan diferencias con estatus socioeconómico, ya que estudiantes en el grupo de menor nivel socioeconómico ( $\mathrm{M}=5.43$, $D E=1)$ manifiestan una menor satisfacción con la vida que los estudiantes del grupo de mayor nivel socioeconómico ( $M=5.9, \mathrm{DE}=0.88)$ (Seligson et al., 2003).

Como herramienta de medición ha demostrado ser útil para evaluar la salud psicológica de niños y adolescentes, ya que se puede obtener información que no puede ser recogida por escalas orientadas hacia lo patológico (Huebner et al., 2003). Además, dada la creciente necesidad de contar con medidas válidas empíricas para el estudio de la satisfacción con la vida de los niños y adolescentes, que sean pertinentes a la edad y que permitan mediciones nacionales y transnacionales (Huebner et al., 2011), esta escala es una alternativa de creciente reconocimiento y estudio en el campo.

El presente estudio, además de recoger estos aprendizajes y fundamentos, busca describir los procedimientos utilizados para la adaptación y aplicación de la BMSLSS, identificando sus propiedades psicométricas en población infantil (entre 10 y 12 años) chilena. 


\section{Método}

\section{Participantes}

La presente investigación forma parte de un estudio internacional más amplio que incluye a 13 países y cuyo propósito general fue indagar en el bienestar psicológico de la población infantil. Este proyecto es liderado por la Sociedad Internacional de Indicadores de la Infancia (The International Society of Child Indicators) y patrocinado por el Fondo de las Naciones Unidas para la Infancia (Unicef).

En esta investigación, participaron 1096 escolares con edades entre 10 y 12 años ( $M=11$ años, $D E$ $=0.89$ ), pertenecientes a tres ciudades de Chile. En la Tabla 1, aparece la información sociodemográfica de la muestra. Se intentó mantener un número balanceado de participantes en todas las categorías, con excepción del tipo de dependencia escolar de los alumnos, en la que se privilegió seleccionar a más alumnos de colegios donde acuden la gran mayoría de escolares. Por otra parte, el procedimiento que se aplicó para la imputación de los datos perdidos fue el algoritmo EM (Expectation-Maximiza- tion). El EM es usado típicamente para computar estimaciones de máxima probabilidad, mediante un procedimiento en muestras que cuentan con datos incompletos (Cuesta, Fonseca-Pedrero, Vallejo, \& Muñiz, 2013).

\section{Instrumentos}

Se empleó la versión en español de la Escala Multidimensional Breve de Satisfacción con la Vida para Estudiantes (BMSLSS). En la aplicación realizada en Chile, la BMSLSS fue traducida del inglés y utilizó una escala de respuesta tipo Likert de 11 puntos $(0=$ totalmente descontento/a; $10=$ totalmente contento/a) (Casas et al., 2011).

Se utilizaron seis preguntas cerradas de autorreporte, que indagan sobre distintos dominios importantes en la vida de los niños, a saber:

Ítem 1: iHasta qué punto te encuentras satisfecho o satisfecha con tu vida familiar?

Ítem 2: iHasta qué punto te encuentras satisfecho o satisfecha con tus amigos y amigas?

Ítem 3: iHasta qué punto te encuentras satisfecho o satisfecha con el barrio donde vives en general?

TABLA 1

Descripción de las variables sociodemográficas de los participantes $(\mathrm{N}=1096)$

\begin{tabular}{lcc}
\hline Variables & $\mathrm{N}$ & $\%$ \\
\hline Sexo & 598 & 54.6 \\
Hombre & 498 & 45.4 \\
Mujer & & \\
Edad & 421 & 38.4 \\
10 años & 217 & 19.8 \\
11 años & 458 & 41.8 \\
12 años & & \\
Región de los escolares & 375 & 34.2 \\
Viña del Mar (V Región) & 425 & 38.8 \\
Concepción (VIII Región) & 296 & 27 \\
Santiago (XIII Región) & & \\
Tipo de dependencia escolar & 633 & 57.8 \\
Municipal & 387 & 35.3 \\
Subvencionados & 76 & 6.9 \\
Particular & & \\
\hline
\end{tabular}

Fuente: elaboración propia 
Ítem 4: ¿Hasta qué punto te encuentras satisfecho o satisfecha con tu experiencia en el colegio?

Ítem 5: ¿Hasta qué punto te encuentras satisfecho o satisfecha contigo mismo/a?

Ítem 6: iHasta qué punto te encuentras satisfecho o satisfecha con toda tu vida en general?

Los ítems fueron promediados para obtener el puntaje total de la BMSLSS que representa la satisfacción general con la vida.

\section{Procedimiento}

Antes del estudio, se realizó una aplicación piloto a una muestra de 290 participantes de 8 colegios (4 de Santiago y 4 de Concepción), de los cuales 4 fueron municipales, 2 subvencionados y 2 particulares. Esto permitió efectuar algunos ajustes a las preguntas, así como estimar el tiempo de respuesta a cada pregunta. El cuestionario fue aplicado por 10 entrevistadores en promedio, en cada región. Se eligieron encuestadores que tuvieran experiencia en la aplicación de encuestas masivas, privilegiándose a personas que contaran con estudios universitarios en ciencias sociales y/o educación.

El cuestionario se aplicó a través de la modalidad de autoadministración, durante el período escolar 2012, en el horario regular de clases. Se cumplieron los protocolos éticos de investigación, con especial énfasis en la confidencialidad de la información producida y el consentimiento informado de los establecimientos educacionales, el asentimiento de los niños y niñas y el consentimiento (pasivo) de sus padres o tutores.

\section{Resultados}

\section{Distribución de las respuestas}

De acuerdo a la Teoría Clásica de los Tests, se examinaron en primer término las características de cada ítem así como del puntaje total de la BMSLSS, con el fin de evaluar la distribución de su normalidad. En las dos primeras columnas de la Tabla 2 se puede apreciar una asimetría negativa con valores entre -2.48 y -1.44 , mientras que los valores de la curtosis oscilaron entre 5.68 y 1.48. La literatura suele mostrar criterios muy diferentes sobre los valores que puedan servir para dar cumplimiento al supuesto de normalidad. En este estudio, se sigue la sugerencia de Kline (2005), quien propone que valores superiores a 3 para la asimetría y 10 para la curtosis deben de ser considerados como indicadores problemáticos de la normalidad. En consecuencia, los resultados informan que todos los ítems cumplen los criterios de normalidad.

En la tercera y cuarta columna de la Tabla 2 se observan los promedios y desviación estándar para los seis ítems de la escala y el puntaje total de la BMSLSS. De manera general, se observa que para cada uno de los ítems, los participantes expresaron una satisfacción cercana al punto máximo de la escala (10) en las diferentes dimensiones evaluadas. Por su parte, el promedio de $8.54(D E=1.57)$ para el puntaje total de la escala BSMLSS muestra que los participantes reportan una alta satisfacción con la vida.

TABLA 2

Asimetría, curtosis, promedios y desviación estándar para los ítems y el puntaje total de la BMSLSS

\begin{tabular}{lcccc}
\hline \multirow{1}{*}{\multicolumn{1}{c}{ Ítems }} & Asimetría & Curtosis & $\mathrm{M}$ & $\mathrm{DE}$ \\
\cline { 2 - 5 } Satisfacción con la familia & -2.48 & 5.68 & 8.88 & 2.33 \\
Satisfacción con amigos/as & -2.08 & 4.13 & 8.7 & 2.26 \\
Satisfacción con el barrio donde vives & -1.52 & 1.48 & 8.1 & 2.73 \\
Satisfacción con el colegio & -1.82 & 2.86 & 8.46 & 2.41 \\
Satisfacción contigo mismo/a & -1.85 & 2.47 & 8.34 & 2.79 \\
Satisfacción con tu vida en general & -2.19 & 4.02 & 8.64 & 2.58 \\
Puntaje total & -1.44 & 2.45 & 8.54 & 1.58 \\
\hline
\end{tabular}

Fuente: elaboración propia 
TABLA 3

Correlaciones entre los ítems y el puntaje total de la BMSLSS

\begin{tabular}{lccccccc}
\hline & 1 & 2 & 3 & 4 & 5 & 6 & 7 \\
\hline 1. Satisfacción con la familia & 1 & & & & & & \\
2. Satisfacción con amigos/as & $0.25^{* *}$ & 1 & & & & & \\
3. Satisfacción con el barrio donde vives & $0.23^{* *}$ & $0.25^{* *}$ & 1 & & & & \\
4. Satisfacción con el colegio & $0.18^{* *}$ & $0.28^{* *}$ & $0.29^{* *}$ & 1 & & & \\
5. Satisfacción contigo mismo/a & $0.2^{* *}$ & $0.19^{* *}$ & $0.24^{* *}$ & $0.24^{* *}$ & 1 & & \\
6.Satisfacción con tu vida en general & $0.28^{* *}$ & $0.22^{* *}$ & $0.38^{* *}$ & $0.27^{* *}$ & $0.57^{* *}$ & 1 & \\
7. Puntaje total & $0.55^{* *}$ & $0.56^{* *}$ & $0.65^{* *}$ & $0.6^{* *}$ & $0.67^{* *}$ & $0.73^{* *}$ & 1 \\
\hline
\end{tabular}

Fuente: elaboración propia

En la Tabla 3, se aprecian las correlaciones entre los ítems y el puntaje total de la BSMLSS. En general, los puntajes de los ítems correlacionaron aceptablemente entre ellos y en la dirección teórica esperada. Asimismo, se analizaron las correlaciones entre cada ítem y la BSMLSS completa, con el objetivo de identificar ítems que no tuvieran asociación con la escala general. Como se observa en la misma Tabla 3, esto no ocurrió, pues el rango de correlaciones entre los seis ítems y la BSMLSS general fue bastante alto (rango entre 0.55 y 0.73 ).

\section{Propiedades psicométricas de la BSMLSS}

Para evaluar la validez de constructo de la escala en el contexto chileno, se aplicó en primer término un análisis factorial exploratorio (AFE) a las respuestas de 311 participantes, seleccionados aleatoriamente, que correspondió aproximadamente al 30\% de la muestra total. Se obtuvo un chi-cuadrado significativo del Test de Esfericidad de Bartlett y un valor del Kaiser-Meyer-Olkin (KMO) igual a
0.74, lo que informa que estos datos son apropiados para realizar este análisis. Para el AFE se utilizó el Método de Ejes Principales, con el fin de obtener la estructura más simple y coherente posible. Los resultados muestran un solo factor que explica el 27 \% de la varianza. En la Tabla 4 se consignan, en la primera columna, las cargas factoriales para cada uno de los ítems, cuyos valores aparecen en un rango entre 0.31 (satisfacción con la familia) y 0.73 (satisfacción con la vida en general).

En la Tabla 4, también se aprecia el índice de dificultad para cada ítem, el cual fue calculado dividiendo el número de escolares que eligieron el punto máximo de la escala (10) por el número total de escolares, en este caso 311. En general, se estima que si la dificultad de un ítem es mayor a 0.75 se trata de un ítem sencillo; en cambio, si la dificultad es inferior a 0.25 , estamos ante un ítem difícil. En este caso, ninguno de los ítems excedió dicho rango. En la tercera columna, se despliegan las correlaciones entre cada ítem y el puntaje total de la escala, sin considerar dicho ítem. Este puntaje corregido es

TABLA 4

Parámetros de confiabilidad para los items de la BMSLSS $(n=311)$

\begin{tabular}{lllll}
\hline & $\begin{array}{l}\text { Carga } \\
\text { Factorial (AFE) }\end{array}$ & $\begin{array}{l}\text { Índice de } \\
\text { Dificultad }\end{array}$ & $\begin{array}{l}\text { Correlación Ítem- } \\
\text { Total Corregida }\end{array}$ & $\begin{array}{l}\text { Alpha si el ítem } \\
\text { fuese eliminado }\end{array}$ \\
\hline Satisfacción con la familia & 0.314 & 0.68 & 0.35 & 0.69 \\
Satisfacción con amigos/as & 0.461 & 0.59 & 0.29 & 0.7 \\
Satisfacción con el barrio donde vives & 0.583 & 0.48 & 0.44 & 0.66 \\
Satisfacción con el colegio & 0.390 & 0.54 & 0.40 & 0.67 \\
Satisfacción contigo mismo/a & 0.528 & 0.46 & 0.52 & 0.63 \\
Satisfacción con tu vida en general & 0.734 & 0.61 & 0.59 & 0.61 \\
\hline
\end{tabular}

Fuente: elaboración propia 
particularmente importante cuando se evalúa una escala con pocos ítems como es el caso de la BMSLSS. Los ítems 5 (satisfacción contigo mismo) y 6 (satisfacción con tu vida en general) correlacionan bien con el puntaje general de la BMSLSS, mientras que el ítem 2 (satisfacción con los amigos) es el que muestra una correlación baja. Finalmente, el alpha de Cronbach de la escala completa fue igual a 0.7 , lo que indica que este constructo tiene una consistencia interna aceptable. Por otra parte, la eliminación de algún ítem no contribuye a mejorar la confiabilidad de la escala.

Con el propósito de confirmar que los seis ítems cargan en una única variable latente, se realizó un análisis factorial confirmatorio (CFA), utilizando el programa estadístico Mplus versión 7.0 (Muthén \& Muthén, 2012). Este CFA fue aplicado al resto de participantes de la muestra $(n=$ 897). Se utilizó el estimador de máxima probabili- dad (ML) y los índices de ajuste revisados fueron: (a) chi cuadrado, (b) Root Mean Square Error of Approximation (RMSEA), (c) Comparative Fit Index of Bentler (CFI), (d) Tucker-Lewis Index (TLI) y (d) Standardized Root Mean SquareResidual (SRMR). Los índices fueron comparados con los parámetros comúnmente aceptados en la literatura (v.g., $>0.9$ para CFI y TLI mientras que $<0.05$ para RMSEA y SRMR). Los valores de los índices de ajuste fueron los siguientes: $\chi^{2}(9)=$ 63.61, $p<0.01$; RMSEA $=0.08$ (90\% de intervalo de confianza $=0.06-0.1)$; CFI $=0.94 ;$ TLI $=0.9$ y SRMR $=0.03$. Estos valores son aceptables, por lo que se puede admitir la estructura única de la BMSLSS. La solución para el CFA aparece en la Figura 1. Como puede observarse, todas las cargas factoriales son altas (rango entre 0.4 y 0.78), valores muy similares a los obtenidos mediante el AFE.

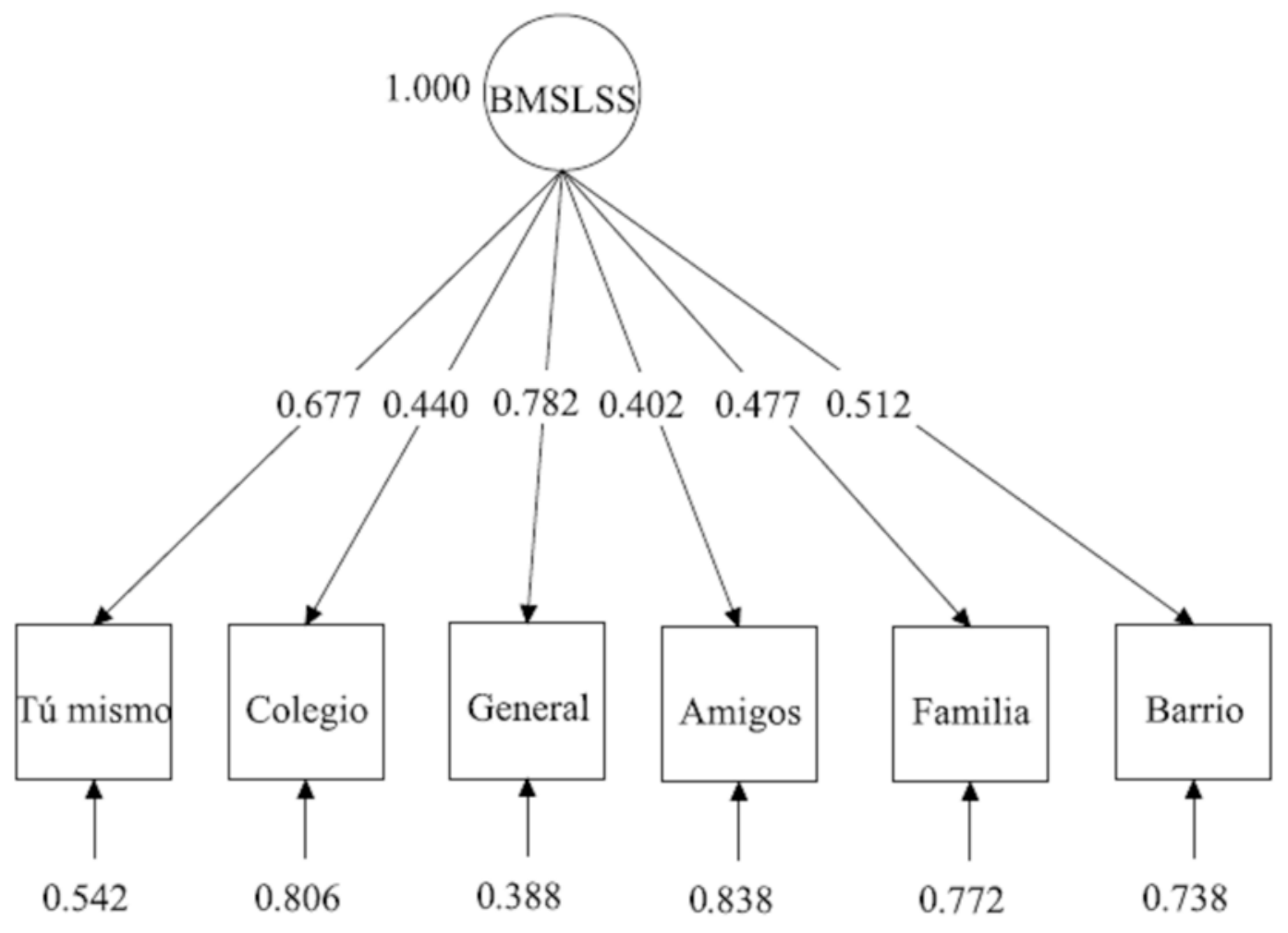

Figura 1. Modelo de un factor de la BSMLSS

Fuente: elaboración propia 
Finalmente, con el propósito de ayudar a la interpretación de los datos, los puntajes de la BMSLSS completa fueron clasificados de acuerdo a los cuartiles 25,50 y 75 . Así, un puntaje superior a 9.83 es considerado una satisfacción alta, mientras que un puntaje inferior a 7.66 se puede describir como de satisfacción baja. Los puntajes que estén entre estos valores pueden ser calificados como de satisfacción moderada con la vida.

\section{Conclusiones y discusión}

El análisis de las propiedades psicométricas de la escala BMSLSS resultantes de la aplicación en niños chilenos de 10, 11 y 12 años están de acuerdo a estudios similares realizados a nivel internacional. Se observa un coeficiente de confiabilidad ligeramente superior en la población infantil estudiada respecto de la validación original, ya que en esta última se obtuvo un resultado de alpha de Cronbach de 0.68 (Seligson et al., 2003), mientras que en Chile fue de 0.7. Esto da cuenta de la estabilidad de los ítems que conforman la escala y de un adecuado nivel de fiabilidad en línea con resultados de estudios internacionales (Huebner et al., 2003; Huebner, Seligson, Valois, \& Suldo, 2006; Seligson et al., 2003; Siyez \& Kaya, 2008; Valois, Paxton, Zullig, \& Huebner, 2006).

En el análisis de correlación entre ítem-puntaje total, aspecto que implica en qué medida el ítem está relacionado con el puntaje total de la escala, muestra una alta correlación con los ítems de satisfacción con el barrio, colegio, consigo mismo y su vida en general. Los dos primeros están vinculados con contextos de socialización secundaria, mientras que los dos últimos se asocian con la evaluación de la propia satisfacción.

Respecto de la estructura factorial de la BSMLSS, los resultados dan cuenta de un solo factor. Esta estructura es corroborada con el AFC en la línea de estudios similares (Huebner et al., 2003).

Dado que los parámetros psicométricos de esta escala son equivalentes a los obtenidos en grupos de edad superior (Seligson et al., 2003), resulta presumible asumir que su adaptación valida el procedimiento de autorreporte en población infantil chilena de 10 a 12 años.
El presente trabajo amplía el repertorio de herramientas de medición psicométrica para la población infantil, adaptando una escala que ha sido validada para adolescentes adecuándola a niños y niñas de menor edad (10, 11 y 12 años), y que además ofrece la posibilidad de establecer comparaciones internacionales en el marco del estudio liderado por la Sociedad Internacional de Indicadores de la Infancia (The International Society of Child Indicators). Esto adquiere particular relevancia en cuanto este estudio ha utilizado una muestra de niños y niñas de una realidad latinoamericana, de un país como Chile en vías de desarrollo y en un grupo de edad en el cual existe baja disponibilidad de instrumental psicométrico adaptado.

Finalmente, este estudio refuerza la idea de incluir la participación sustantiva de los niños y niñas en la medición de satisfacción con la vida, y la consideración de un abordaje desde la perspectiva del niño, ya que evalúa sus respuestas como fundamentales y no basadas en las percepciones de los adultos sobre su bienestar. Ambos aspectos dan cuenta de la sintonía de este estudio con la Convención Internacional de los Derechos de los Niños y Niñas, manteniendo un eje relevante de estudios similares realizados en otros países, según lo referido con anterioridad.

Respecto de las limitaciones del estudio, el presente tuvo un foco en la población escolar urbana, ya que fueron aplicadas en el contexto de una investigación internacional solo a las tres ciudades con mayor concentración demográfica de Chile (Santiago, Concepción y Valparaíso), lo que deja afuera criterios de inclusión como etnia, ruralidad y otras variables que podrían surgir de un muestreo más diverso que el realizado en esta investigación, por lo que sus resultados no podrían generalizarse para poblaciones distintas del muestreo indicado.

\section{Referencias}

Aguado, L. (2009). Un índice de no consumo de alimentos en la niñez para Colombia. Revista Latinoamericana de Ciencias Sociales, Niñez y Juventud, 7(2), 735-760. 
Alfaro, J., Valdenegro, B., \& Oyarzún, D. (2013). Análisis de propiedades psicométricas del Índice de Bienestar Personal en una muestra de adolescentes chilenos. Diversitas: Perspectivas en Psicología, 9(1), 13-27.

Ben-Arieh, A. (2008). The child indicators movement: Past, present and future. Child Indicators Research, 1(1), 3-16.

Bradshaw, J., \& Lau, M. (2010). Child well-being in the Pacific Rim. Child Indicators Research, 3(3), 367-383.

Bradshaw, J., \& Richarson, D. (2009). An index of child well-being in Europe. Child Indicators Research, 2(3), 319-351.

Campbell, A., Converse, P., \& Rodgers, W. (1976). The quality of American life: Perceptions, evaluations and satisfactions. Nueva York: Russell Sage Foundation.

Casas, F. (2010a). El bienestar personal: su investigación en la infancia y la adolescencia. Encuentros en Psicología Social, 5(1), 85-101.

Casas, F. (2010b). Indicadores sociales subjetivos y bienestar en la infancia y adolescencia. En Propuesta de un sistema de indicadores sobre bienestar infantil en España (pp. 34-51). Madrid: Unicef.

Casas, F., Sarriera, J. C., Abs, D., Coenders, G., Alfaro, J., Saforcada, E., \& Tonon, G. (2011). Subjective indicators of personal well-being among adolescents. Performance and results for different scales in Latin-language speaking countries: A contribution to the international debate. Child Indicators Research, 5(1), 1-28.

Casas, F., Sarriera, J. C., Alfaro, J., González, M., Malo, S., Bertrán, I., ... Valdenegro, B. (2012). Testing the Personal Wellbeing Index on 12-16 year-old adolescents in 3 different countries with 2 new items. Social Indicators Research, 105(3), 461-482.

Cuesta, M., Fonseca-Pedrero, E., Vallejo, G., \& Muñiz, J. (2013). Datos perdidos y propiedades psicométricas en los tests de personalidad. Anales de Psicología, 29(1), 285-292.

Cummins, R. A., Eckersley, R., Van Pallant, J., Van Vugt, J., \& Misajon, R. (2003). Developing a national index of subjective wellbeing: The Australian Unity Wellbeing Index. Social Indicators Research, 64(2), 159-190.
Diener, E. (1984). Subjective wellbeing. Psychological Bulletin, 95(3), 542-575.

Diener, E. (2006). Guidelines for national indicators of subjective well-being and ill-being. Applied Research in Quality of Life, 1(2), 151-157.

Diener, E., \& Suh, E. (1997). Measuring quality of live: Economic, social, and subjetive indicators. Social Indicators Research, 40(1-2), 189-216.

Figuer, C., Casas, F., Buxarrais, M., González, M., Tey, A., Noguera, E., \& Rodríguez, J. (2004). Los valores y su influencia en la satisfacción vital de los adolescentes entre los 12 y los 16 años: estudio de algunos correlatos. Apuntes de Psicología, 22(1), 3-23.

Gademann, A. M., Schonert-Reichl, K. A., \& Zumbo, B. D. (2010). Investigating validity evidence of the Satisfaction with Life Scale adapted for children. Social Indicators Research, 96(2), 229-247.

Gallardo Cuadra, I., \& Moyano Díaz, E. (2012). Análisis psicométrico de las escalas Ryff (versión española) en una muestra de adolescentes chilenos. Universitas Psychologica, 11(3), 931-939.

Huebner, E. S. (1991). Initial development of the Student's Life Satisfaction Scale. School Psychology International, 12(3), 231-240.

Huebner, E. S. (2004). Research on assessment of life satisfaction of children and adolescents. Social Indicators Research, 66(1-2), 3-33.

Huebner, E. S., Antaramian, S. P., Hills, K. J., Lewis, A. D., \& Saha, R. (2011). Stability and predictive validity of the Brief Multidimensional Students' Life Satisfaction Scale. Child Indicators Research, 4(1), 161-168.

Huebner, E. S., \& Gilman, R. (2002). An introduction to the Multidimensional Students' Life Satisfaction Scale. Social Indicators Research, 60(1-3), 115-122.

Huebner, E. S., Seligson, J. L., Valois, R. F., \& Suldo, S. M. (2006). A Review of the Brief Multidimensional Students' Life Satisfaction Scale. Social Indicators Research, 79(3), 477-484.

Huebner, E. S., Suldo, S. M., \& Valois, R. F. (13 de marzo de 2003). Psychometric Properties of Two Brief Measures of Children's Life Satisfaction: The Students' Life Satisfaction Scale (SLSS) and the Brief Multidimensional Students' Life Satisfaction Scale (BMSLSS). Trabajo presentado en la conferencia Indicators of Positive Development Conference, Washington. 
Disponible en http://www.childtrends.org/wp-content/uploads/2013/05/Child_Trends-2003_03_12_ PD_PDConfHSVP.pdf

Huebner, E. S., Suldo, S. M., Valois, R. F., \& Drane, J. W. (2006). The Brief Multidimensional Students' Life Satisfaction Scale: Sex, race, and grade effects for applications with middle school students. Applied Research in Quality of Life, 1(2), 211-216.

Jiang, X., Huebner, E. S., \& Hills, K. (2013). Parent attachment and early adolescents' life satisfaction: The mediating effect of hope. Psychology in the Schools, 50(4), 340-352.

Kline, R. B. (2005). Principles and practice of structural equation modeling (2.a ed.). Nueva York: The Guilford Press.

Lau, A. L. D., Cummins, R. A., \& McPherson, W. (2005). An investigation into the cross-cultural equivalence of the Personal Wellbeing Index. Social Indicators Research, 72, 403-432.

McKnight, C. G., Huebner, E. S., \& Suldo, S. (2002). Relationships among stressful life events, temperament, problem behavior, and global life satisfaction in adolescents. Psychology in the Schools, 39(6), 677-687.

Moyano Díaz, E., Flores Moraga, E., \& Soromaa, H. (2011). Fiabilidad y validez de constructo del test MUNSH para medir felicidad en población de adultos mayores chilenos. Universitas Psychologica, $10(2), 567-580$.

Muthén, L. K., \& Muthén, B. O. (2012). Mplus user's guide. Los Angeles, CA: Muthén \& Muthén.

Pavot, W., \& Diener, E. (1993). Review of the Satisfaction with Life Scale. Psychological Assessment, 5(2), 164-172.

Paxton, R. J., Valois, R. F., Huebner, E. S., \& Drane, J. W. (2006). Opportunity for adult bonding/meaningful neighborhood roles and life-satisfaction among USA middle school students. Social Indicators Research, 79(2), 291-312.

Petito, F., \& Cummins, R. A. (2000). Quality of life in adolescence: The role of perceived control, parenting style, and social support. Behaviour Change, 17(3), 196-207.

Pollard, E. L., \& Lee, P. D. (2003). Child wellbeing: A systematic review of the literature. Social Indicators Research, 61(1), 59-78.
Puente Díaz, R., \& Cavazos Arroyo, J. (2013). Personality factors, affect, and autonomy support as predictors of life satisfaction. Universitas Psychologica, 12(1), 41-53.

Seligson, J. L., Huebner, E. S., \& Valois, R. F. (2003). Preliminary validation of the Brief Multidimensional Students' Life Satisfaction Scale (BMSLSS). Social Indicators Research, 61(2), 121-145.

Seligson, J. L., Huebner, E. S., \& Valois, R. F. (2005). An investigation of a Brief Life Satisfaction Scale with elementary school children. Social Indicators Research, 73(3), 355-374.

Siyez, D. M., \& Kaya, A. (2008). Validity and reliability of the Brief Multidimensional Students' Life Satisfaction Scale with Turkish children. Journal of Psychoeducational Assessment, 26(2), 139-147.

Tiliouine, H., Cummins, R., \& Davern, M. (2006). Measuring wellbeing in developing countries: The case of Algeria. Social Indicators Research, 75(1), 1-30.

Tomyn, A. J., \& Cummins, R. A. (2011). The subjective wellbeing of high-school students: Validating the Personal Wellbeing Index-School Children. Social Indicators Research, 101(3), 405-418.

Valois, R. F., Paxton, R. J., Zullig, K. J., \& Huebner, E. S. (2006). Life Satisfaction and Violent Behaviors among Middle School Students. Journal of Child and Family Studies, 15(6), 695-707.

Valois, R. F., Zullig, K. J., Huebner, E. S., \& Drane, J. W. (2003). Dieting behaviors, weight perceptions, and life satisfaction among public high school adolescents. Eating Disorders, 11(4), 271-88.

Valois, R. F., Zullig, K. J., Huebner, E. S., \& Drane, J. W. (2004a). Life satisfaction and suicide among high school adolescents. Social Indicators Research, 66(1-2), 81-105.

Valois, R. F., Zullig, K. J., Huebner, E. S., \& Drane, J. W. (2004b). Physical activity behaviors and perceived life satisfaction among public high school adolescents. Journal School of Health, 74(2), 59-65.

Veenhoven, R. (1994). El estudio de la satisfacción con la vida. Intervención Psicosocial, 3(9), 87-116.

Veenhoven, R. (2002). Why social policy needs subjective indicators. Social Indicators Research, 58(1), 33-46.

Vera-Villarroel, P., Urzúa, A., Pavez, P., Celis-Atenas, K., \& Silva, J. (2012). Evaluation of subjective well- 


\section{Propiedades psicométricas de la Escala Breve Multidimensional \\ DE SATISFACCiÓN CON LA VIdA PARA EstUdiantes}

being : Analysis of the Satisfaction With Life Scale in Chilean population. Universitas Psychologica, 11(3), 719-727.

Webb, D. (2009). Subjective wellbeing on the Tibetan plateau: An exploratory investigation. Journal of Happiness Studies, 10(6), 753-768.
Yiengprugsawan, V., Seubsman, S., Khamman, S., \& Lim, L. L. (2010). Personal Wellbeing Index in a national cohort of 87,134 Thai adults. Social Indicators Research, 98(2), 201-215. 
